\title{
CONCEPTUALISER UNE RECHERCHE EN PSYCHANALYSE
} Observation et empathie. Écriture sublimée, écriture d'une passion Catherine Cyssau

\section{Association Recherches en psychanalyse | «Recherches en psychanalyse »}

2004/1 $\mathrm{n}^{0} 1 \mid$ pages 131 à 144

ISSN $1767-5448$

ISBN 2847950303

Article disponible en ligne à l'adresse :

http://www.cairn.info/revue-recherches-en-psychanalyse-2004-1-page-131.htm

\section{Pour citer cet article :}

Catherine Cyssau, « Conceptualiser une recherche en psychanalyse. Observation et empathie. Écriture sublimée, écriture d'une passion », Recherches en psychanalyse 2004/1 ( $\left.\mathrm{n}^{\circ} 1\right)$, p. 131-144.

DOI 10.3917/rep.001.0131

Distribution électronique Cairn.info pour Association Recherches en psychanalyse.

(C) Association Recherches en psychanalyse. Tous droits réservés pour tous pays.

La reproduction ou représentation de cet article, notamment par photocopie, n'est autorisée que dans les limites des conditions générales d'utilisation du site ou, le cas échéant, des conditions générales de la licence souscrite par votre établissement. Toute autre reproduction ou représentation, en tout ou partie, sous quelque forme et de quelque manière que ce soit, est interdite sauf accord préalable et écrit de l'éditeur, en dehors des cas prévus par la législation en vigueur en France. Il est précisé que son stockage dans une base de données est également interdit. 


\title{
Conceptualiser une recherche en psychanalyse. Observation et empathie. Écriture sublimée, écriture d'une passion
}

\author{
Catherine Cyssau
}

\section{L'OBSERVATION ET LA MÉTHODE}

Nous conviendrons, à la suite de Freud, que l'observation du fait clinique n'est pas séparable de la méthode utilisée: la méthode en psychanalyse est déterminante d'une spécificité clinique. Freud insiste sur le caractère erroné que prendrait une évaluation des résultats, en dehors d'une prise en compte du procédé de leur découverte, méthode qu'il estime être « irremplaçable pour des fins scientifiques » : «On ne peut contester valablement les résultats auxquels conduit cette méthode breuérienne modifiée, si on laisse la méthode de côté et si l'on utilise uniquement la méthode habituelle de l'examen des malades ${ }^{1}{ }^{»}$.

Les voies d'approche d'une psychopathologie psychanalytique ne feront pas l'économie du «traitement psychique» ${ }^{2}$. Car seule la méthode analytique conçoit les conditions de réception intrinsèque permettant à l'œuvre de théorie d'évoluer à partir du déplacement des obstacles issus de la clinique qui est inférée par la méthode. Il n'y a de clinique et de psychopathologie psychanalytique qu'à travers le dispositif de la situation analytique qui n'est lui-même pas dissociable des investissements associatifs du transfert dans la parole ${ }^{3}$.

Freud ajoute que l'observation des processus psychiques a cette particularité de ne pas être extérieure aux processus psychiques de l'observation. L'appareil psychique est objet observé et outil d'observation clinique, en même

1. S. Freud (1896), «Sur l'étiologie de l'hystérie», in Euvres Complètes Psychanalyse III, Paris, P.U.F., 1989, p. 180.

2. S. Freud (1890), «Traitement psychique. Traitement d'âme», in Résultats, idées, problèmes I, Paris, P.U.F., p. 2-23.

3. P. Fédida, «Psychopathologie générale et psychopathologie fondamentale», in Crise et contre-transfert, Paris, P.U.F., 1992, p. 293. 
temps qu'il conçoit le lieu fictif de la construction métapsychologique. Cette communauté fondamentale est fondatrice de la théorie psychanalytique. La triple fonction des processus psychiques dispose le transfert à être le nouage technique, à la fois l'outil d'observation et de transmission des matériaux psychiques, et le mode d'engendrement des formations mnésiques propres à l'appareil psychique lui-même.

La recherche en psychanalyse va interroger son rapport à la clinique d'une part, la clinique issue du «traitement psychique» déterminé par le référent analytique. Celui-ci a modifié l'observation clinique en une clinique de l'écoute qui privilégie des éléments de présentation indirecte sur lesquels l'attention du patient et de l'analyste n'est pas dirigée. Elle remplace la visibilité sémiologique d'une vue synthétique de la maladie visée par la clinique psychiatrique. Le postulat de l'inconscient préfère la fragmentation à la synthèse, et n'hésite pas à associer le hasard au transfert, privilégiant de la clinique une vue processuelle fragmentaire, discontinue.

\section{L'OUTIL DU TRANSFERT}

La méthode de Freud engage un mouvement évolutif de théorisation qui peut être comparable aux modes d'investissements associatifs de la mémoire, au jeu indéterminé entre résistance et transfert, refoulement et retour du refoulé. Il revient au transfert d'animer les détails d'une mémoire associative qui n'a pas un déroulement linéaire direct de la source au but, mais, suivant la voie du détour pulsionnel, relève d'un espace intermédiaire qui fait jouer le processus où s'incarne chaque résurgence du matériau psychique, selon sa coloration actuelle nécessairement ancienne et nouvelle. Ce mouvement discontinu, fragmentaire de la découverte psychique va caractériser les voies de progression de la recherche en psychopathologie laquelle se dirige par diffraction et dissémination plus que par accumulation et généralisation ${ }^{4}$. Pris dans le mouvement d'une recherche, le concept en psychanalyse se forme par association de traces mnésiques et perceptives. Celles-ci, à chaque fois qu'elles s'animent, se modifient, se déplacent et changent d'aspect.

Pour la théorie psychanalytique, le concept vise surtout à éviter sa fixation sous forme d'un «fuero», «loi espagnole ancienne restée en vigueur» ${ }^{5}$ visant le maintien de privilèges révolus dans certaines provinces. Mais la vivacité du concept, en cours de théorisation, ouvert au déplacement de son transfert, ne doit pas faire perdre de vue la permanence où il s'inscrit, également, comme

4. P. Fédida, P. Lacoste, «Psychopathologie/Métapsychologie », in Revue Internationale de Psychopathologie, $\mathrm{n}^{\circ}$ 8, Paris, P.U.F., 1992, p. 599.

5. S. Freud, «Lettre à Fliess du 6-12-1896», in La naissance de la psychanalyse, Paris, P.U.F., 1979, p. 156. 
découverte pour perdurer. La théorie psychanalytique freudienne demeure comme la mémoire de l'inconscient, indestructible et toujours actuelle, bien qu'elle soit cependant évolutive et mobile.

Le respect épistémologique de la méthode et des concepts fondateurs de la métapsychologie conduit à rendre compte des nouveaux obstacles produits dans la rencontre clinique; ceux-ci comportent une vivacité méthodologique propice à une exploration innovante de la psychopathologie. Là où Freud historiquement est parti de l'observation psychopathologique pour construire une métapsychologie de l'appareil psychique, nous disposons désormais d'une théorie métapsychologique fondatrice de la méthode clinique depuis laquelle laisser la psychopathologie se modifier à travers la méthode analytique ; et ceci non seulement dans le mouvement d'un traitement psychanalytique ou psychothérapique, mais dans l'œuvre de théorisation qui va s'y associer.

\section{LA RÉGULATION DU CONTRE-TRANSFERT ET LA PERLABORATION}

La recherche en psychanalyse ouvre de nouvelles voies de théorisations pour la psychopathologie. Elle ne le peut, toutefois, que si l'on tient compte d'une régulation par le contre-transfert au contact du transfert. Le transfert comme méthode d'observation permet de faire émerger la notion de contretransfert.

L'instrument de perception du contre-transfert contribue au travail de perlaboration. Il contre l'esprit de synthèse du moi et les risques réductionnistes qu'il comporte pour la situation analytique et une position de recherche clinique. Le contre-transfert est un «principe de régulation dépressive» ${ }^{6}$ qui assure une distinction et une dissymétrie entre la scène psychique de l'analyste et celle du patient. Il est aussi l'instrument qui préserve le chercheur, comme l'analyste, des risques de confusion entre l'objet de recherche et la personne du chercheur.

Cette perception endopsychique par le préconscient du «senti inconscient» de l'analyste, sous la suggestion du transfert, est ce qui évite à l'analyste de réagir, en retour, par l'usage de la suggestion à l'influence que lui fait subir, psychiquement, chaque patient au cours des séances successives du jour. Il ne s'agit pas d'un évitement défensif. L'influence du transfert du patient touche aux points aveugles, aux lignes de résistances qui nervurent, à ce moment-là de sa vie, la psyché de l'analyste. Mais, que la résistance de l'analyste soit

6. P. Fédida, «Le contre-transfert en question», in Crise et contre-transfert, p. 198: «Le contre-transfert reçoit (...) une fonction de régulation harmonique de la réception des dissonances et ainsi de l'analyse des contraires. L'économie qu'il réalise obéit au principe de laisser les dissonances se porter d'elles-mêmes à leur résolution au lieu de vouloir les réduire synthétiquement en leur imposant une règle de consonance.» 
appelée à recevoir les transferts du patient laisse envisager que l'inanalysé renvoie, chez l'analyste ou le chercheur, à quelque chose de plus originaire que le refoulé, quelque chose qui a eu lieu mais n'a pas encore été vécu, dirait Winnicott. Il s'agit donc d'une découverte psychique innovante pour l'analyste. Cette découverte peut devenir, à son tour, l'instrument d'une recherche théorique féconde. Autrement dit, la perlaboration contre-transférentielle est nécessaire à l'élaboration théorique d'une recherche psychanalytique qui soit liée par une psychopathologie clinique. La suggestion du transfert a pour effet associatif, sur la psyché de l'analyste, de mobiliser des traces mnésiques de perception. Elles sont d'une nature pré-inconsciente ${ }^{7}$ et leur inscription ne relève pas des refoulements. Ces traces de l'amnésie originaire viennent être actualisées depuis les sollicitations du transfert, elles trouvent un destin psychique du devenir conscient suivant la voie directe de l'affect, sous forme d'intuitions théorisantes. Il n'est pas alors question d'un retour du refoulé. Peutêtre, s'agit-il du travail culturel qui s'offre comme voie de sublimation avec l'accès à la sexualité génitale ${ }^{8}$.

L'analyste modifié sous l'influence des transferts du jour trouve, par la régulation du contre-transfert, un recours à l'Einfühlung. Elle transite par les modifications de sa psyché où se perlabore le détour de l'interprétation. L'Einfühlung conçoit la résonance du côté des mouvements perlaboratifs de résistance et de dissonance chez l'analyste soumis aux suggestions des transferts. Il est question de pouvoir penser ce qui, dans la régulation économique des transferts par le contre-transfert, va transformer ${ }^{9}$ la réaction suggestive initiale aux effets suggestifs des transferts, en un lieu d'identification primaire. Celui-ci fonde le contre-transfert à l'oblique et dissymétriquement. L'empathie n'est pas, en ce cas, un dérivé mimétique de l'identification secondaire et elle n'est pas de l'ordre de la compréhension. Selon Pierre Fédida, «l'intérêt de concevoir le contre-transfert comme procédant du complexe du père non seulement évite de le penser comme pure et simple réaction dans la relation du patient à lui, mais tendrait surtout à lui accorder une fonction archaïque [acte fondateur] propre à réévaluer la nature du phénomène de transfert ${ }^{10}{ }_{\text {» }}$.

Mettre en perspective transfert et suggestion, contre-transfert et identification primaire positionne une interaction asymptotique de leur nouage. Le mouvement de l'identification primaire pour le contre-transfert n'induit pas un forçage du mouvement transférentiel d'un retour résistant du refoulé, comme c'est le cas dans la technique active de Ferenczi ou dans la suggestion hypno-

7. Cf. C. Cyssau, (1995) Au lieu du geste, Paris, P.U.F.; «L'intranquillité d'aimer», in S. Le Poulichet, Les addictions, Monographies de psychopathologie, Paris, P.U.F, 2000 ; et Les dépressions de la vie, Paris, P.U.F., 2004.

8. S. Freud (1914), «Pour introduire le narcissisme», in La vie sexuelle, Paris, P.U.F., 1977. 327.

9. Cf. P. Fédida, «Topiques de la théorie », in L'absence, Paris, Gallimard, 1978, pp. 326-

10. P. Fédida, «Le contre-transfert en question », in Crise et contre-transfert, op. cit., pp. 196197. 
tique du «traitement d'âme », à ses débuts pour Freud. Mais il va supporter que la courbe du transfert, pour effectuer son trajet, ne le puisse qu'en asymptote au réglage du contre-transfert. L'incitation transférentielle relève des capacités contre-transférentielles de perlaboration aux points de résistance d'un actuel inanalysable, variant chez l'analyste, tel qu'il axe la courbure transférentielle. C'est la dissonance du «point de surdité» ${ }^{11}$ de l'analyste qui, en se perlaborant, soutient et engendre le mouvement du transfert et la construction, via l'actuel, des détours de la pulsion par le refoulé, l'infantile, ou l'originaire.

\section{LE LIEN D’UNE THÉORIE DE LA CLINIQUE}

La figure résistante du symptôme est alors ce qui fait lien, c'est-à-dire tiercéise, conflictualise, métaphorise la dualité d'une relation du transfert au contre-transfert. C'est également dans ce rapport de l'analyste à l'identification primaire que réside la possibilité, pour la construction, de tenir ensemble une "vérité de croyance » et la réalité psychique du penser ${ }^{12}$.

Pour le chercheur, ce passage du croire au penser reste déterminant d'une spécificité des recherches en psychanalyse ${ }^{13}$. La pensée psychanalytique a pour fondement scientifique de reposer sur l'observation clinique psychopathologique. Les idées qu'une telle recherche va ensuite développer «ne constituent pas les fondations mais le faîte de tout l'édifice, et elles peuvent sans dommage être remplacées et enlevées », estime Freud, en $1914{ }^{14}$.

De cette remarque, regardons déjà le second point. Il concerne la nature évolutive que Freud octroyait à ses conceptualisations. Elle veut éviter que puisse se voir alloué à la discipline tout caractère illusoirement religieux ou idéologique émanant d'une psychanalyse de Freud entièrement achevée et inamovible. La théorie psychanalytique de Freud appelle, au contraire, à la reconstruction et au chantier renouvelé du «faîte de l'édifice » psychanalytique.

Le premier point aborde, quant à lui, les conditions des fondements scientifiques d'une théorie psychanalytique. L'observation, définie par la méthode analytique, est le lieu d'expérience des « intuitions fondamentales » qui forment le noyau conceptuel de la psychanalyse, de façon «à peine moins discutable», dit Freud, que le sont, pour la physique, «ses intuitions fondamentales sur la matière, les centres de force, l'attraction, etc.».

L'intuition correspond à l'empathie «flottante», laquelle laisse se produire, selon J.L. Nancy, «des résonances qui ne vont pas se former à l'unisson de

11. Nous empruntons la notion à J.M. Vives, «Pulsions invocante et destins de la voix, Recherches cliniques en psychanalyse », in Psychologie Clinique, $\mathrm{n}^{\circ}$ 13, 2002, pp. 79-92.

12. Cf. C. Cyssau, «La construction du père dans la clinique des cas limites », in $\mathrm{J}$. André, L'oubli du père, Paris, P.U.F., 2004 (à paraître).

13. P. Fédida, L'absence, Paris, Gallimard, 1978, p. 326.

14. S. Freud (1914), Pour introduire le narcissisme, op. cit., p. 85. 
l'émission... mais contre elle, à son contact, c'est-à-dire au plus près mais peutêtre au plus loin en même temps, en une sorte d'écho inversé, ou bien selon un rapport lui-même (sexuel ou pas ?) incommensurable. Que me fait-on entendre? À quoi veut-on que je prête l'oreille? Vers quoi, de quel côté faut-il la dresser, cette oreille? $\gg^{15}$. L'intuition théorique se forge au contact de l'observation, à l'écoute psychopathologique contre le transfert, c'est-à-dire en résonance empathique à celui-ci. Elle définit un rapport de désir, de nature sexuelle, à la fois le Wunsch freudien du vœu œdipien et l'appétit de désir de Descartes ou de Hume (J.L. Nancy) ${ }^{16}$.

La vérité de croyance comporte, ainsi, la réalité psychique du penser. Cette démarche scientifique ne l'est pourtant qu'à se conceptualiser depuis l'entre deux du transfert et du contre-transfert, de l'écriture et de la lecture, de l'émission et de l'entente. Il y faut le concours de deux " actions psychiques» ${ }^{17}$ qui, en rapport sexuel de transfert, vont être capables de convertir le croire en un acte de penser effectuant une théorie de la clinique.

Le passage du croire au penser accepte la mort de l'individu et renonce à l'immortalité de la substance chimique ou de la pensée à travers l'espèce. Nous touchons là au point-limite que constitue le rapport phylogénétique au biologique, dans l'assise scientifique de la théorie psychanalytique de Freud. Il y aurait une immortalité de la substance ou des pulsions qui, certes, assujettit l'individu à l'espèce mais avec un certain profit pour le moi et le narcissisme, stigmatisé par "His majesty the baby» ${ }^{18}$.

En l'absence de deux actions psychiques distinctes et liées, le croire rencontre l'écueil de l'auto-observation délirante, à partir de la projection. Freud distingue, selon ce modèle, le système de penser philosophique où l'introspection épouse les conditions de projection du délire d'auto-observation. Le système de penser de la psychanalyse, à l'opposé, est fondé d'un rapport d'observation. Celui-ci émane des deux scènes psychiques séparées, de leur tension d'écartement et de contact. À cet entre deux, circulent les fondements d'une conceptualisation qui ne se limite pas à 1'auto-observation, sans renoncer aux sources sexuelles du désir dont s'alimente encore le fait de penser.

\section{ÉCRITURE, SYMPTÔME, SUBLIMATION ET PASSION}

Conceptualiser une recherche en psychanalyse perlabore un chemin de l'intuition clinique qui relève, à la fois, de la sublimation et de «la passion

15. J.-L. Nancy, L'«il y $a » d u$ rapport sexuel, Paris, Gallilée, 2001, p. 10.

16. Ibid., p. 38 .

17. S. Freud (1914), Pour introduire le narcissisme, op. cit., p. 84.

18. Ibid., pp. 85-86, 96. Nous ne développons pas davantage ce point-ci. 
amoureuse» des perversions. L'œuvre d'écriture du conceptuel n'est pas abstraite du fait clinique, ni absoute du fait sexuel. L'écriture, qui forme une recherche psychanalytique ayant un destin d'accomplissement, évite doublement l'écueil du refoulement: en satisfaisant, d'une part, au titre de la sublimation, l'idéal du moi, et d'autre part, comme résultante de la passion et de l'amour, en satisfaisant «l'idéal sexuel» 19 .

Sans l'apport de ce double mouvement de passion et de sublimation, l'introspection philosophique menace les théories de la psychanalyse, d'idéologie ou de religion. Auto-engendrement, auto-observation, auto-érotisme, auto-satisfaction déploient une conviction délirante et échouent à métaphoriser l'altération hypocondriaque que la conversion de l'affect en penser appelle à faire, au sens d'acte.

Il y a réinterprétation par l'écriture du travail de l'analyste. Il existe un fonctionnement métaphorique des concepts dont le symptôme est le paradigme. L'écriture, à ce titre, est constitutive du centre de l'absence selon P. Fédida, soit de l'acte métaphorique. Tel est le mouvement ouvert de la théorie dans la recherche psychanalytique. C'est aussi ce qui distingue métapsychologie et métaphysique. La métaphore est existentielle à l'écriture du théorique et non plus une figure rhétorique. La métaphore existentielle suppose le temps gestuel qui est la dynamique du transfert. On est alors aux antipodes de la recherche psychologique «anti-métaphorique » explicative, sans remous. La passion est ce qui anime la théorie perlaborative d'une recherche en psychanalyse. Il n'existe, pour cette recherche, aucune vérification, laquelle viserait à « soustraire du concept la métaphore ${ }^{20}$ qui est l'œuvre de transfert.

La résistance du symptôme détermine encore la fonction métaphorique de l'écriture. L'écriture d'un transfert à la théorie met en jeu une position « métatransférentielle» ${ }^{21}$, celle-ci relève du réglage contre-transférentiel. Les conditions méta-transférentielles garantissent que la recherche en psychanalyse ne se contente pas de l'animisme de la certitude croyante qui lie la métaphysique à la psychose schizophrénique ou soutient l'interprétation du paranoïaque. L'écriture renvoie à la double inscription d'une trace, support d'un destin métaphorique possible. Elle réalise un enregistrement à l'identique de ce qui a été silencieusement vécu psychiquement par l'analyste au contact de la clinique du transfert, sa répétition exacte semble être la transposition, le déplacement nécessaire à l'existence d'une trace transférable. De plus, l'écriture générée par un mouvement de perlaboration transférentielle réalise le mode d'élaboration par la théorie. De ces deux mouvements intrinsèques de l'écriture -inscription et élaboration - attendus d'une recherche en psychanalyse, la métaphoricité du symptôme est le ferment.

19. S. Freud (1914), Pour introduire le narcissisme, op. cit., p. 104.

20. P. Fédida (1978), L’absence, op . cit., p. 318.

21. Ibid. 


\section{ACTUALITÉ MÉTAPHORIQUE DU TRANSFERT}

Freud s'est, d'emblée confronté à la psychopathologie des névroses actuelles, et il n'a pas laissé hors champ de sa pratique analytique les fameux états limites qui conçoivent une sorte d'actualité contemporaine de la clinique du sujet moderne. Il n'est qu'à citer l'analyse de trois mois d'Hilda Doolittle en 1933, poursuivie trois autres mois en 1934. De cette première étape analytique, Hilda Doolittle nous livre la transgression précieuse de ses notes de séances, au jour le jour. Sa résistance à l'analyse, ses vœux de mort à l'égard de Freud, son incapacité à se laisser aller à l'association libre, alors même que poète, elle écrit le mot à mot de ses séances, ont inspiré à Freud ces deux remarques cliniques sur la technique analytique : l'intérêt de la patiente pour les objets de Freud, pour le lieu de son bureau, voire pour son animal favori lui fait tenir ses propos qu'écrit Hilda Doolittle: «Vous êtes la seule personne qui ait jamais pénétré dans cette pièce et regardé les objets avant de me regarder» ${ }^{22}$.

Il y a dans l'émergence du sexuel une part de fortuit qui tient à la coalescence du hasard et du transfert, à leur rencontre imprévisible. Les transferts utilisant la complaisance d'un facteur fortuit disposent d'un choix de traduction, ils réalisent «des éditions revues et corrigées », une sublimation leur offre «de devenir conscients en s'étayant sur une particularité réelle, habilement utilisée, de la personne du médecin ou des circonstances qui l'entourent ${ }^{23}$ ». L'actualité fortuite représente une voie de dérivation pour l'amnésie infantile, et elle est parfois la seule voie. C'est à l'actuel que la construction doit d'être croyante, mais c'est à la réalité psychique primitive qu'elle ranime qu'elle doit sa vérité.

L'intérêt transférentiel de Doolittle pour les objets et les lieux des morts revient-il à absentifier la personne de l'analyste pour être la seule personne présente ? Cela n'est pas sans évoquer la dimension narcissique de l'identification projective. La fin de l'analyse est convoquée dans son actualité d'angoisse comme coïncidence avec le dénouement du transfert, du fait de l'âge, de l'impuissance, de l'abstinence. Mais, sous l'impuissance de vieillesse de Freud, de quelle impuissance psychique ${ }^{24}$ serait-il, en même temps, question pour Doolittle? Quelle serait la fixation infantile qui rend, en ce cas, l'analyse trop actuelle?

Bien avant Lacan ${ }^{25}$, Freud faisait ce constat : «En analyse, la personne est morte quand l'analyse est terminée.» «Aujourd'hui, raconte Hilda Doolittle,

22. H. Doolittle (1974), Écrit sur le mur, H.D. visage de Freud, trad. de l'américain par F. de Gruson, Paris, Denoël, 1977, p. 215.

23. S. Freud, Fragment d'une analyse d'hystérie (Dora), (1905), Cinq psychanalyses, Paris, P.U.F., 1975, p. 87.

24. S. Freud, (1912) «Du rabaissement généralisé de la vie amoureuse», in O.C., Psychanalyse, t. XI, Paris, P.U.F.

25. J. Lacan, Variantes de la cure type, Écrits, Paris, Seuil, 1966. 
quand je suis rentrée dans son cabinet, le Professeur m'a dit : «J'étais en train de penser à ce que vous m'avez dit, que cela ne vaut pas la peine d'aimer un vieil homme de soixante-dix-sept ans.» Je n'avais rien dit de tel et le lui fis remarquer. Il sourit, de ce sourire ironique, en coin : «Je n'ai pas dit que cela ne valait pas la peine, j'ai dit que j'avais peur», dis-je. Mais il me plongea dans le trouble en disant: «En analyse, la personne est morte quand l'analyse est terminée.» Quelle personne? «Cela ne ferait aucune différence si j'avais soixante-dix-sept ou quarante-sept ans », dit-il ${ }^{26}$.

Lors de la séance précédente, Hilda Doolittle a parlé d'un rêve. Dans ce rêve, Havelock Ellis avec sa barbe blanche remplace Freud, mais il ne s'intéresse vraiment pas à la psychanalyse, elle va donc l'ennuyer. Il lui revient alors la peur que lui causa un accident mystérieux de son père, la vue du sang qu'il suscita, dont elle ne veut pas parler à Freud, puis l'oubli de cet événement pendant trente-cinq ans. Dans un retour à la réalité actuelle, Hilda Doolittle a cette réaction à l'interprétation que lui donne Freud de sa résistance à l'analyse : Elle pense et l'écrit: «Mieux vaut une analyse ratée ou «retardée » que de laisser voir [au Professeur] la réelle terreur que m'inspire la menace latente des nazis ${ }^{27}{ }^{2}$.

N'est-ce pas là une différence notable, avec les névroses de transfert, des névroses actuelles de transfert que produisent déjà au cours d'une analyse les états limites ${ }^{28}$ ? La mémoire s'y trouve différemment habitée, investie et construite. Ainsi, le texte d'Hilda Doolittle rencontre et annonce les «Constructions dans l'analyse», que Freud publie en 1937 : en ce cas, seul le souvenir de couverture surgit, avec sa charge sensible et affectée, alors que le refoulé peut demeurer ce centre amnésique de l'absence ${ }^{29}$.

L'ennui pressenti de l'analyste, ce manque de plaisir à l'activité psychique du transfert, du fait de son âge avancé, ferait craindre à Doolittle de ne pas savoir solliciter ses capacités métaphoriques. L'impuissance psychique projetée sur l'analyste devient l'actualité qui vectorise le transfert. Ce dernier sollicite,

26. H. Doolittle (1974), L'avent, H.D. visage de Freud, trad. de l'américain par F. de Gruson, Paris, Denoël, 1977, pp. 58-59.

27. Ibid., pp. 56-57.

28. Cf. sur cette distinction, notre article «La mesure de l'acte en psychothérapie», in Psychologie clinique, Recherches cliniques en psychanalyse, $\mathrm{n}^{\circ} 13,2002$, pp. 229-240.

29. S. Freud, «Constructions dans l'analyse», in Résultats, idées, problèmes II, Paris, P.U.F., 1937, pp. 269-281: «Ce qui m’a frappé» écrit Freud, «dans quelques analyses, c'est que la communication d'une construction manifestement pertinente provoquait chez les analysés un phénomène surprenant et d'abord incompréhensible. Ils sentaient émerger des souvenirs très vivaces, qu'ils qualifiaient eux-mêmes d' «excessivement nets »; ils retrouvaient cependant, non pas l'événement même qui était le contenu de la construction, mais des détails voisins de ce contenu, par exemple, avec une extrême précision, les visages des personnes qui y figuraient, ou les pièces dans lesquelles quelque chose de semblable aurait pu se passer, ou bien encore, de façon un peu plus éloignée, les objets contenus dans ces pièces et que la construction ne pouvait évidemment pas connaître. Cela se produisait aussi bien dans les rêves survenant immédiatement après la communication, que dans des états de rêveries diurnes ». 
justement, un point aveugle bien connu chez Freud, celui de la blessure et de la vue du sang ${ }^{30}$. La résistance muée en ennui contre la suggestion du transfert influence le retour œdipien du souvenir refoulé, celui d'un mystérieux accident du père de Doolittle. Pourtant, ce souvenir ne saurait faire retour sans la contingence fortuite de l'affect de peur. La terreur nazie ravive l'angoisse à la vue du sang de la blessure du père. Cette fois, la menace de danger porte sur l'objet de la fausse connexion transférentielle, Freud lui-même. Le désir d'inceste est un désir pour la jeune fille d'être possédée et déflorée par son père. La vue du sang a convié, chez Doolittle, à la fois ce désir et le ressentiment qu'éprouve encore une femme confrontée à la déception d'avoir perdu, avec l'hymen, l'investissement d'organe dont il était doté pour elle ${ }^{31}$. Cette dépossession engendre son hostilité et ses vœux de mort que la vue du sang attribué au père, accidentellement blessé réveille encore. Un double mouvement œdipien se reporte depuis l'homme qui l'a possédée sexuellement en la déflorant, d'une part sur le père (qui l'a « donnée » à un autre homme) et d'autre part sur la mère (qui l'a si mal dotée). L'acuité perceptive du transfert placée devant le danger d'une terreur bien réelle et qui se porte, ici, sur l'actualité de la menace nazie, donne une consistance de réalité au danger de réel qui existe lorsqu'à la puberté, le rapport sexuel retrouve les vœux d'inceste et de meurtre de l'Edipe noués à la réalité d'acte.

«Quand je lui ai dit un jour que le temps passait trop vite (le sentait-il ou ne le sentait-il pas?), il prit une attitude à demi comique, il étendit le bras comme s'il s'adressait avec ironie à une présence ou à un auditoire imaginaires. Le temps, dit-il. Il prononça le mot de cette manière ambiguë, inimitable qui était la sienne... Dans ce seul mot, il paraissait accumuler un ensemble d'émotions contradictoires ; il y avait de l'ironie, de la prière, de la défiance auxquelles s'ajoutait une sorte de pathos vague et tendre. On eut dit que le mot contenait une charge excessive, une sorte d'explosif qui à tout moment pouvait éclater. (En un sens, beaucoup de ses mots explosaient effectivement, détruisaient des prisons, des barrages et des digues inutiles, provoquant des éboulements c'est vrai, mais ouvrant en même temps des mines de trésors cachés.) Le temps, ditil de nouveau, cette fois plus paisiblement, et il ajouta: Le temps galope.» ${ }^{32}$

L'impuissance du temps semble la corde raide où vient danser son amour du vieil homme. Ce n'est pas le fantasme, en ce cas, qui habille le désir, mais

30. Cf. les très beaux textes de M. Schneider sur l'analyse freudienne de ce point.

31. Dans «Le tabou de la virginité » (1918), Freud prête à l'hymen, un statut psychique d'organe, pour la fille, lui conférant une équivalence avec l'organe masculin du pénis, O.C., psychanalyse, Paris, P.U.F., t. 15, pp. 77-96.

32. H. Doolittle, «Écrit sur le mur», in H.D. visage de Freud, op. cit., 1974, p. 192. Cette remarque de Freud transmise par sa patiente, m'évoque Le temps presse écrit par Antonio Tabucchi. Sous forme d'une pièce de théâtre composée d'un long monologue, il est question d'un dialogue avec un mort, au lit du mort dans une chambre d'hôpital, entre un frère et son frère venant de mourir. 
l'émotion dans ses sources actuelles de réalité. Le temps de la vie et de son âge, le temps de la mort et le temps venu du dialogue avec le/les morts conduisent à repenser, également, comment l'écriture transgressive de Doolittle construit transférentiellement le contre-transfert qui devient, en ce cas, une création inédite du transfert du patient. Cette création subjective semble faire face à l'enjeu sexuel du temps menaçant le pouvoir d'injection identificatoire du narcissisme, et face aux énigmes anachroniques du meurtre et de l'inceste, restées familières, par intense fixation infantile, dans la psychopathologie actuelle des formations limites de la psychopathologie.

Mais cette création, qui enclôt le contre-transfert au sein du transfert, correspond aussi avec l'enjeu des transferts interminables. L'imposture indentificatoire du récit de cure, auquel se livre Doolittle, n'a-t-elle pas pour conséquence de symétriser les scènes psychiques du transfert et du contretransfert? L'écriture tiendrait-elle lieu d'un transfert latéral, écriture éprise d'une analyse de l'analyste, où l'immortalité d'une telle emprise rendrait la fin des transferts évanescente et le temps aveugle de ses meurtres? Il faudrait, à ce propos, se demander si la passion des retrouvailles avec les morts transgénérationnels ne fait pas partie de ce fantasme d'éternité où s'éternisent des analyses interminables, ainsi que des recherches dont l'idéal maintient le projet d'inachèvement en l'état tout puissant si tenace de son impuissance psychique.

«J'aurais voulu prendre le sablier dans ma main et le renverser de manière que les sables de sa vie eussent autant d'années à parcourir vers l'avant qu'ils en avaient déjà franchis jusqu'ici... Je voulais échanger mes années contre les siennes... Mettez H.D. à la place de Sigmund Freud... Et cela peut se faire. Quelqu'un déjà le fit et s'offrit à le faire dans une pièce. C'était une pièce grecque, n'est-ce pas ?... La pièce ne s'appelait-elle pas Alceste? Je me demande. Et bien sûr, l'un de ces trois auteurs doit l'avoir écrite - ils sont là en haut de la bibliothèque du Professeur, à droite de la double porte largement ouverte qui mène à son cabinet Privé. Eschyle? Sophocle? Euripide? Qui écrivit l'Alceste? Mais l'auteur n'importe pas vraiment, car la pièce se joue maintenant - en tout cas nous sommes en train de la jouer, le vieux Professeur et moi. Le Professeur y joue deux rôles. Il est Hercule livrant un combat à la Mort et il est l'amant sur le point de mourir. Et en outre, lui-même, dans son propre personnage a fait revivre les morts, et surgir d'une tombe vivante nombre d'enfants morts ou mourants ${ }^{33} \gg$.

Si l'on pense que le contre-transfert intervient avec la disparition de l'analyste en personne, et si l'on pense qu'une telle perception endopsychique est ce qui suscite la production, la traduction métaphorique des transferts, ne sommes-nous pas poussée à interroger le soubassement fantasmatique du concept scientifique d'état-limite ? Le temps et le sexuel y sont moins anachroniquement fantasmatiques qu'affectés d'une angoisse sensitive dont le réalisme,

33. Ibid., pp. 191-192. 
voire l'hyperréalisme diurne, propulse la rupture et le changement, au même titre que le souvenir de couverture pour la construction. Le lieu au contraire, représenté par la bibliothèque de Freud, son chien, ses objets helléniques, et le cadastre des immortels éternels classés sur les rayonnages, ainsi que le texte du journal clinique qu'écrit Hilda Doolittle après chaque séance, forment l'enjeu d'une continuité qui semble cependant nécessaire à la transcription d'une actualité de la construction, qu'il ne faut probablement pas confondre avec la transmission.

\section{SPÉCIFICITÉ DU CHERCHEUR EN PSYCHANALYSE}

De même que l'événement du cas avait pu m'apparaître relever de la fonction du transfert, l'événement de la recherche en psychanalyse est épistémologiquement référé au réglage du contre-transfert. L'écriture de la théorie devient l'inscription et le chemin élaboratif de la métaphore. La puissance métaphorique d'une recherche est à considérer du point de vue de sa capacité empathique d'altérisation ${ }^{34}$ : pouvoir entendre l'autre de l'autre se situe de la façon la plus éloignée avec l'analogique et l'imitatif du même, que l'identification compréhensive risque toujours de signifier. L'élaboration d'une métapsychologie du symptôme ne saurait ainsi dissocier le concept ni de la métaphore, ni du sexuel de la pulsion. L'une et l'autre le fondent indissociablement à être le lieu de nomination d'une vérité psychique qui n'est pas scientifiquement ou psychologiquement reproductible dans l'esprit de sa vérification.

Le contre-transfert est ce qui peut permettre de tenir ensemble la proposition selon laquelle la psychopathologie en psychanalyse ne peut adopter ni la méthode d'exploration du modèle psychiatrique, ni celle issue des neurosciences ou encore de la biologie ou de l'anthropologie, avec cette deuxième proposition selon laquelle le matériel recensé dans ces divers champs extrinsèques au domaine analytique peuvent venir interpeller l'œuvre de théorie analytique. Il ne s'agit pas alors d'importer une méthode extrinsèque à la psychanalyse pour l'objectiver, ainsi qu'un courant de la psychologie ou de la psychiatrie neuro-comportementaliste psychiatrique peut rêver une hégémonie totalitaire du fait psychologique.

Mais il n'est pas non plus question d'interdisciplinarité entre deux champs. L'interdisciplinarité développe une conception analogique de la métaphore, celle-ci construit peut-être davantage un dispositif psychique en miroir qu'un espace de saisissement et de découverte. Dans la situation analytique, l'appareil psychique étant à la fois l'observé et l'observant supprime la voie analogique.

34. P. Lacoue Labarthe, J.-L. Nancy, «La panique politique», in Cahiers Confrontation, $\mathrm{n}^{\circ} 2$, 1979, Paris, Aubier, pp. 33-58. 
La recherche en psychanalyse n'est pas enceinte dans le baquet de la cure et cependant, la formulation de J. Laplanche poursuit une certaine efficience ${ }^{35}$. Mais comment procède-t-elle? La construction de P. Fédida, se situe du côté d'une «transcendance du contre-transfert». Lorsque le psychanalyste, chercheur à l'université, se met au contact de théorisations ou d'un matériel clinique extrinsèque à son champ propre, son approche sera celle d'une résonance contretransférentielle (Einfühlung) qui vient solliciter une métaphore du transfert, celle-ci conçoit la continuité en évitant la menace totalisante du moi. Le passage méthodologique, que régit l'outil contre-transférentiel, vectorise une spécificité du chercheur en psychanalyse à l'université et rend son approche de phénomènes de société, de données anthropologiques, d'œuvres d'art, de biologie, de géologie, de paléontologie, d'archéologie, de mathématiques, etc., ordonnée d'une vue propre à sa qualité.

\section{Catherine CYSSAU Maître de conférences HDR à l'UFR de Sciences humaines cliniques Équipe interne «Le traitement psychique» 6 impasse Marie Blanche 75018 Paris}

\footnotetext{
Catherine Cyssau - Conceptualiser une recherche en psychanalyse. Observation et empathie. Écriture sublimée, écriture d'une passion.
}

Résumé : Dans la recherche en psychanalyse, nous faisons déprendre l'observation du transfert. Celui-ci ne devient un outil que dès lors qu'il permet de faire émerger la notion de contre-transfert. Nous avançons l'idée que la recherche en psychanalyse est épistémologiquement déterminée par le réglage du contre-transfert. La psychopathologie en psychanalyse constitue le foyer évolutif de la clinique. À partir d'elle, surgissent des points de renouvellement et de décentrement des théories du symptôme. C'est la singularité du cas clinique qui fait évoluer la perspective psychopathologique. À travers le récit clinique par Hilda Doolittle de sa cure avec S. Freud, nous nous attachons à la potentialité transformative des fonctionnements limites de la psychanalyse depuis leur résistance à la méthode. Cette « fiction » de Doolitttle nous conduit à questionner la fonction de l'écriture dans l'élaboration théorique et clinique. Nous soutenons que les concepts psychopathologiques se voient réinterprétés par la métaphorisation d'une écriture qui est parataxique du phénomène qu'elle tend à saisir.

35. J. Laplanche, Le baquet. Transcendance du transfert, Problématiques V, Paris, P.U.F., 1987. 
Mots-clés: Méthode - Ecriture - Contre-transfert - Conceptualisation - Métaphore Névroses actuelles.

Catherine Cyssau - Conceptualising Research into Psychoanalysis. Observation or Empathy. Sublimated Writing or the Writing of Passion

Summary : In research into psychoanalysis, we have a certain detachment towards the observation of transference. The latter only becomes a useful tool when the notion of countertransference is looked at. This article puts forward the idea that research into psychoanalysis is epistemologically determined by the organisation of counter-transference. Psychopathology in psychoanalysis represents the evolving heart of clinical practice. From there, points of renewal and the decentralising of theories of the symptom break through at the surface. It is the unique nature of each clinical case that helps the psychopathological perspective to evolve. Through the clinical narratives of Hilda Doolittle and her cure with Freud, we will look closely at the transformation potential of the border functionings of psychoanalysis as from their resistance to this method. The 'narrative fiction' of Doolittle leads us to wonder about the function of writing in the elaboration of theory and clinical practice. This article maintains that psychopathological concepts are reinterpreted through the metaphorical conceptualisation of writing that is parataxic with the phenomenon that is aims at seizing hold of.

Key-words: Method - Writing - Counter-transference - Conceptualisation - Metaphor Actual nevrosis. 